\title{
Qualidade de vida de pacientes com Diabetes mellitus na Atenção Primária
}

\section{Quality of life of patients with Diabetes mellitus in Primary Care}

Calidad de vida de los pacientes con Diabetes mellitus en Atención Primaria

Victor Guilherme Pereira da Silva Marques ORCID: https://orcid.org/0000-0002-7441-9811 Centro Universitário do Piauí, Brasil

E-mail: guilhermevictor521@gmail.com

Eveline Bittencourt Barreto

ORCID: https://orcid.org/0000-0002-2720-1801

Universidade Federal de Juiz de Fora, Brasil

E-mail: eveline.bittencourt@hotmail.com

Célio Pereira de Sousa Júnior

ORCID: https://orcid.org/0000-0003-0726-0668 Universidade Federal do Pará, Brasil

E-mail: academicocelio@gmail.com

Mariel Wágner Holanda Lima

ORCID: https://orcid.org/0000-0001-5063-3891

Secretaria de educação do Estado do Rio Grande do Norte, Brasil

E-mail: marielhoolanda@gmail.com

Ana Carla Almeida de Melo

ORCID: https://orcid.org/0000-0001-5853-8899

Universidade Potiguar, Brasil

E-mail: anacarla_melo@yahoo.com.br

Marks Passos Santos

ORCID: https://orcid.org/0000-0003-1180-404X Faculdade AGES de Medicina, Brasil

E-mail: enfer.marks@hotmail.com

Millena Raimunda Martins de Almeida Carvalho

ORCID: https://orcid.org/0000-0003-2431-2431 Universidade Federal do Piauí, Brasil

E-mail: millenamartinsalmeida2@gmail.com

Roberto de Sousa Costa

ORCID: https://orcid.org/0000-0003-0285-8409

Centro Universitário Santo Agostinho, Brasil

E-mail: robertocosta.enf@gmail.com

Estefany de Jesus Silva

ORCID: https://orcid.org/0000-0001-8849-759X

Centro Universitário Santo Agostinho, Brasil

E-mail: estefanyjc19@gmail.com

Roseane Oliveira Veras

ORCID: https://orcid.org/0000-0003-2746-1475

Faculdade Mauricio de Nassau - Parnaíba, Brasil

E-mail: roseaneoliveira2@outlook.com

Larissa de Lima Machado Bandeira

ORCID: https://orcid.org/0000-0001-5486-9914

Estácio de Teresina, Brasil

E-mail: larissabandeiraphb@gmail.com

Laisa Garcia Matos

ORCID: https://orcid.org/0000-0002-7137-5585

Christus Faculdade do Piauí, Brasil

E-mail: laisagarciamatos45@gmail.com

Eryson Lira da Silva

ORCID: https://orcid.org/0000-0003-1188-5249

Centro Universitário Unifacid Wyden, Brasil

E-mail: erysonlira@gmail.com

\section{Resumo}

O presente estudo tem como objetivo realizar um levantamento bibliográfico acerca da qualidade de vida de pacientes com diabetes mellitus na atenção primária. Trata-se de uma revisão integrativa da literatura, que fez as buscas na Biblioteca Virtual em Saúde (BVS), com o auxílio das bases de dados Literatura Latino-Americana e do Caribe em Ciências da Saúde (LILACS), Scientific Electronic Library Online (SciELO), Base de Dados de Enfermagem (BDENF) 
e PUBMED. Os artigos foram coletados no período de janeiro a fevereiro de 2021. Foram utilizados os descritores: Atenção primária à saúde, Diabetes mellitus, Educação em saúde, Promoção da saúde e Qualidade de vida. Após a aplicação dos critérios de inclusão, restaram 25 estudos. Diversos meios são utilizados para a redução de complicações graves aos pacientes com diabetes, como: tratamento medicamentoso, atividade física dentre outros meios que possam fazer com que esse paciente tenha uma boa qualidade de vida, que não afete tanto sua rotina.

Palavras-chave: Atenção primária à saúde; Diabetes mellitus; Educação em saúde; Promoção da saúde; Qualidade de vida.

\begin{abstract}
The present study aims to conduct a literature survey about the quality of life of patients with diabetes mellitus in primary care. This is an integrative literature review, which searched the Virtual Health Library (VHL), with the help of the databases Latin American and Caribbean Literature on Health Sciences (LILACS), Scientific Electronic Library Online (SciELO), Nursing Database (BDENF) and PUBMED. The articles were collected from January to February 2021. The following descriptors were used: Primary health care, Diabetes mellitus, Health education, Health promotion, and Quality of life. After applying the inclusion criteria, 25 studies remained. Several means are used to reduce serious complications to patients with diabetes, such as drug treatment, physical activity, and other means that can make this patient have a good quality of life that does not affect so much his routine.
\end{abstract}

Keywords: Primary health care; Diabetes mellitus; Health education; Health promotion; Quality of life.

\title{
Resumen
}

Este estudio pretende realizar un estudio bibliográfico sobre la calidad de vida de los pacientes con diabetes mellitus en atención primaria. Se trata de una revisión bibliográfica integradora, que realizó las búsquedas en la Biblioteca Virtual en Salud (BVS), con el auxilio de las bases de datos Latin American and Caribbean Literature on Health Sciences (LILACS), Scientific Electronic Library Online (SciELO), Nursing Database (BDENF) y PUBMED. Los artículos se recogieron de enero a febrero de 2021. Se utilizaron los siguientes descriptores: Atención primaria de salud, Diabetes mellitus, Educación sanitaria, Promoción de la salud y Calidad de vida. Tras aplicar los criterios de inclusión, quedaron 25 estudios. Se utilizan varios medios para reducir las complicaciones graves a los pacientes con diabetes, tales como: tratamiento farmacológico, actividad física entre otros medios que pueden hacer que este paciente tenga una buena calidad de vida que no afecte tanto su rutina.

Palabras clave: Atención primaria de salud; Diabetes mellitus; Educación sanitaria; Promoción de la salud; Calidad de vida.

\section{Introdução}

O Sistema Único de Saúde (SUS) se encaminha para a sua consolidação por meio do Pacto pela Saúde, que aponta como prioridade, entre outras, a qualificação da atenção primária, definindo como modelo de atenção à saúde no Brasil a Estratégia Saúde da Família. A atenção primária à saúde é definida como estratégia de organização do sistema de saúde para realizar ações de promoção à saúde, prevenção de agravos, diagnóstico, tratamento de problemas de saúde mais prevalentes e reabilitação individual e coletiva. A atenção primária à saúde é a porta de entrada do sistema de saúde, que lida com desafios especiais, pois muitos dos problemas trazidos pelos pacientes são geralmente vagos e não relacionados a doenças orgânicas específicas (Azevedo et al., 2013).

O diabetes mellitus tipo 2 (DM2) é uma doença caracterizada por hiperglicemia crônica, decorrente da produção diminuída ou ausente de insulina pelo pâncreas e/ou pela resistência periférica à ação desse hormônio. Entre os tipos existentes de diabetes, o DM2 responsabiliza-se por, aproximadamente, 90 a 95\% de todos os casos da doença. O número de pacientes com diabetes em todo o mundo, no ano de 2015, foi estimado em 415 milhões pela Federação Internacional de Diabetes1 e a projeção é que esse número aumente para 673 milhões até 2040. O Brasil ocupa o quarto lugar no mundo em número de casos de diabetes, com 14,3 milhões de pacientes com a doença (Assunção et al., 2017).

Diante desses números, é importante que sejam investigados as complicações agudas e crônicas do DM e sua relação com o tempo de diagnóstico. As complicações agudas incluem a hipoglicemia, o estado hiperglicêmico hiperosmolar e a cetoacidose diabética. Já as crônicas incluem a retinopatia, nefropatia, cardiopatia isquêmica, neuropatias, doença cerebrovascular e vascular periférica. As degenerativas mais frequentes são o infarto agudo do miocárdio, a arteriopatia periférica, o acidente vascular cerebral e a microangiopatia (Cortez et al., 2015). 
No Brasil, o Ministério da Saúde, criou vários programas de controles de doenças de maior impacto na população, e no caso do diabetes mellitus, foi criado em 2002 o Programa Nacional de Hipertensão e Diabetes Mellitus - Hiperdia, na tentativa de reorientar a assistência farmacêutica para o fornecimento contínuo do medicamento, assim como monitoramento das condições clínicas dos usuários dos serviços de saúde (Cortez et al., 2015).

A educação em saúde para o manejo e a prevenção das complicações crônicas do DM na Atenção primária à saúde (APS) abarca diversos sistemas que se encontram em constante interação, entre eles os profissionais em si, as equipes de Saúde da Família (EqSFs), as equipes do Núcleo de Apoio à Saúde da Família (NASF), a gestão local, a gestão municipal, a estrutura local, as políticas de saúde; em um dinâmico processo que permite a formação de redes dentro de redes. A tessitura impressa na complexidade das redes envolve constantes relações marcadas por intensas interligações, inter-relações, interconexões e múltiplas possibilidades oferecidas por esses sistemas, numa visão integradora que seja capaz de conceber inúmeras possibilidades (Salci, Meirelles \& Silva, 2018).

A modificação de estilo de vida, necessária após o diagnóstico de DM, é um desafio que abrange não só o tratamento da doença, mas outros aspectos relacionados diretamente com a qualidade de vida. A aceitação de ser portador de uma doença crônica, que exige um tratamento contínuo, impacta emocionalmente de maneira negativa, gera frustrações e estresse que dificultam a aderência a um tratamento contínuo. A adesão a novas rotinas nutricionais, a prática de exercício físico e ao tratamento medicamentoso são atitudes que dependem de conhecimento e entendimento da doença, requerem capacidade de enfrentamento para os ajustes necessários para a manutenção de um bom controle metabólico. Assim, a educação na autogestão do diabetes mellitus é um fator determinante para o manejo da doença. A correta administração do cuidado à saúde exige um complexo regime comportamental, que inclui mudanças de atitudes que contribuem de forma significativa os atributos do autocuidado. Essas medidas melhoram de maneira direta a qualidade de vida do indivíduo, e podem ser ensinadas por profissionais de saúde e aprendidas pelos indivíduos através de programas que incentivem o autocuidado afim de melhorar as suas condições de manejo da doença (Vietta et al., 2019).

Nesse contexto, o paciente com DM, em particular tipo 2 enfrenta diversas dificuldades de ajustamento, as quais podem afetar a apreciação subjetiva que faz de sua condição de vida, de acordo com o estágio de desenvolvimento das complicações relacionadas à doença. Além disso, é uma condição crônica que persiste por toda a vida e que, por vezes, vem acompanhada de outras comorbidades. As complicações agudas, decorrentes do DM, também exercem impacto direto sobre a qualidade de vida (QV), pois aumentam a predisposição a transtornos depressivos e de ansiedade, interferem nas relações de trabalho, no desempenho de tarefas domiciliares e escolares, bem como na própria independência (Faria et al.,2013).

No geral, pode-se perceber que o significado de qualidade de vida inclui amplas variedades de condições internas e externas escolhidas por cada um de nós. Percebe-se, então, a importância de pesquisas que definam quais as principais dificuldades do paciente diabético que irão influenciam na sua qualidade de vida. Uma vez elaborado esses dados, eles serão uteis para auxiliar na melhoria dos serviços de saúde (Frota, Guedes \& Lopes, 2015).

Pesquisas revelam que a QV de pacientes com DM é menor do que naqueles sem a doença, e os aspectos envolvidos nessa relação ainda não são totalmente conhecidos. Cabe ressaltar que algumas variáveis tais como: tipo de DM, uso de insulina, idade, complicações, nível social, fatores psicológicos, etnias, educação, conhecimento sobre a doença, tipo de assistência, entre outras, pode interferir na QV destes pacientes (Corrêa et al., 2017).

Ao não cumprir o regime medicamentoso com Antidiabéticos Orais (Aos) o paciente com DM 2 não obtém os benefícios fisiológicos esperados (normoglicemia), deteriora a relação paciente/profissional de saúde, além de elevar os custos médicos com a gênese de complicações micro e macrovasculares, como infarto agudo do miocárdio, acidente vascular encefálico, insuficiência renal, retinopatias, amputações e óbitos diretos (Araújo et al., 2011). 
Para obtenção de um bom controle metabólico, é preponderante que os profissionais de saúde supervisionem a adesão ao plano terapêutico estabelecido e também considerem as adversidades que o paciente encontra em seu cotidiano para o manejo da doença, sabendo respeitar seus limites e explorar suas possibilidades. Para tanto, o acesso à educação em saúde é uma condição essencial para que o paciente possa elaborar o processo de doença e construir um espaço para conhecer sua doença e encontrar novas e melhores maneiras de lidar com as vicissitudes do tratamento (Zulian et al., 2013).

Neste sendo, a principal fonte de apoio às pessoas com DM advém das Estratégias Saúde da Família (ESF) que, desde a sua incorporação na APS, reorganizam o modelo curativista tradicional, em ascensão na década de 80, para um modelo baseado na clínica ampliada. A equipe de ESF estabelece o vínculo profissional-usuário por meio de práticas de educação em saúde e intervenções de acompanhamento das pessoas com DM (Moreschi et al., 2018).

O presente estudo tem como objetivo realizar um levantamento bibliográfico acerca da qualidade de vida de pacientes com diabetes mellitus na atenção primária.

\section{Metodologia}

Trata-se de um estudo descritivo, do tipo revisão integrativa de literatura, de caráter qualitativo uma vez que é definida como um tipo de investigação voltada para o aspecto qualitativo de uma determinada questão, nesse caso, a importância da qualidade de vida de pacientes com diabetes mellitus na atenção primária. A revisão de literatura permite aprofundar dentro de diversos autores e referenciais, sobre os discursos e principais temas abordados (Pereira et al., 2018).

Para direcionar a presente revisão delineou-se como questão norteadora: "O que a literatura aborda sobre a qualidade de vida de pacientes com diabetes mellitus na atenção primária?".

Para a construção deste trabalho, a busca dos artigos foi realizada na Biblioteca Virtual em Saúde (BVS), com o auxílio das bases de dados Literatura Latino-Americana e do Caribe em Ciências da Saúde (LILACS), Scientific Electronic Library Online (SciELO), Base de Dados de Enfermagem (BDENF) e PUBMED. Os artigos foram coletados no período de janeiro a fevereiro de 2021. Foram utilizados os descritores: "Atenção primária à saúde", "Diabetes mellitus", "Educação em saúde", "Promoção da saúde" e "Qualidade de vida", localizados na lista dos Descritores em Ciências da Saúde.

Foram selecionados como critérios de inclusão: artigos completos disponíveis integralmente nas bases de dados elencadas, em idiomas português, espanhol e inglês, com o recorte temporal de 2011 a 2021 e relacionados com a temática. Foram excluídos artigos duplicados, incompletos, resumos, resenhas, debates, artigos publicados em anais de eventos e indisponíveis na íntegra.

\section{Resultados e Discussão}

A partir da revisão de literatura e análise dos estudos indexados nas bases de dados eletrônicas, acerca da temática proposta, foram encontrados 250 estudos científicos, sendo que, apenas 100 estudos foram selecionados, 55 atenderam aos critérios de inclusão previamente estabelecidos, destes, 30 foram excluídos com base nos critérios de exclusão, restando 25 artigos para composição e análise do estudo. O fluxograma com o detalhamento das etapas de pesquisa está apresentado a seguir na Figura 1. 
Figura 1. Fluxograma de identificação e seleção dos artigos.2021.

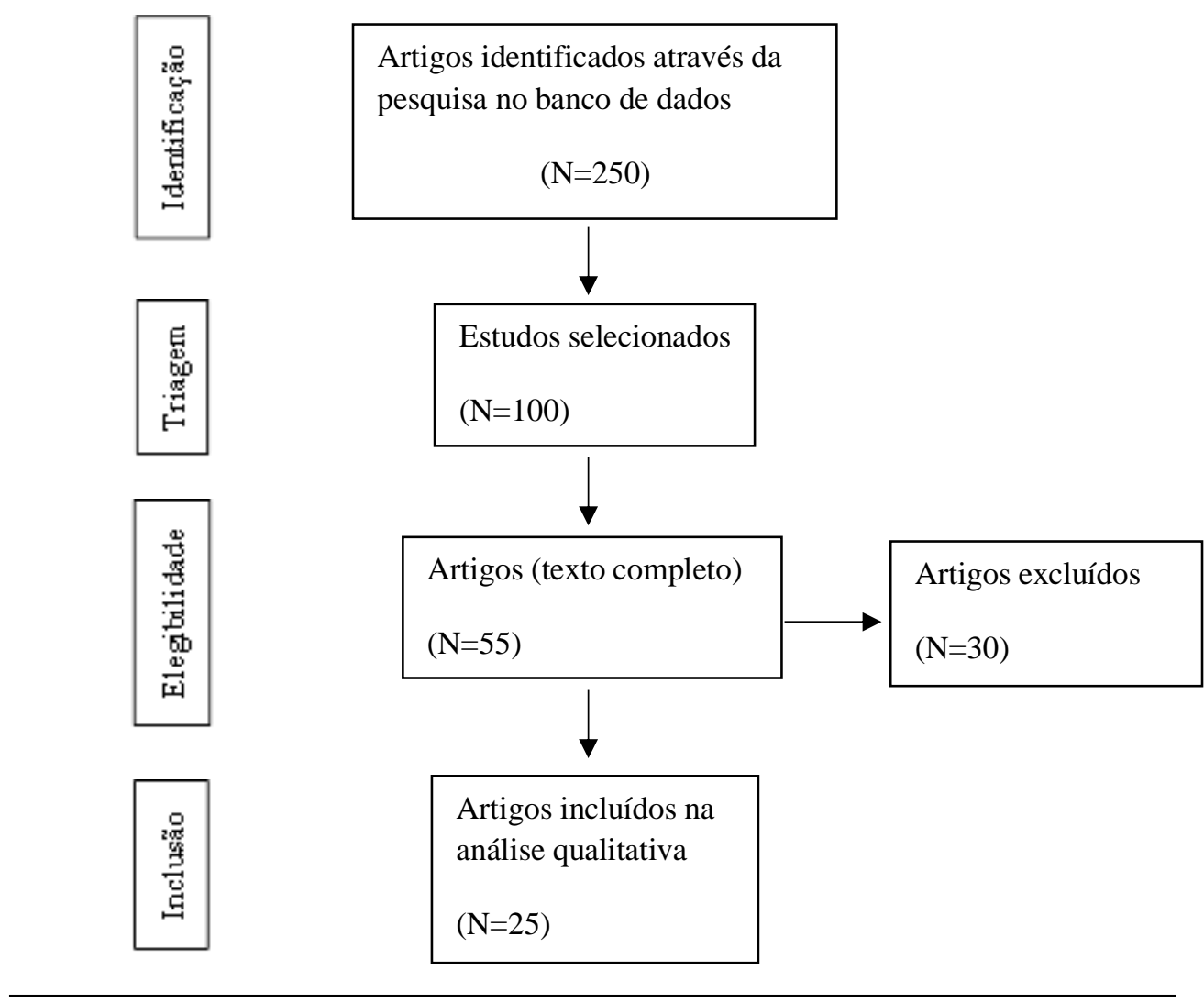

Fonte: Autores (2021).

Para análise, interpretação, discussão e elaboração dos dados, foram explorados 20 estudos compreendidos entre os anos 2011 a 2021, apresentando os fatores que levam a qualidade de vida de pacientes com diabetes mellitus na atenção primária.

As pessoas que sofrem de diferentes doenças crônicas têm de aprender a conviver com várias limitações em seu cotidiano, uma vez que a doença exige adaptação do paciente e dos familiares em diferentes aspectos da vida, e geralmente tais mudanças se desdobram com o tempo (Azevedo et al., 2013).

A manutenção do controle metabólico satisfatório garante ao diabético uma redução no risco dessas complicações. Para que isso ocorra, é necessário que esses indivíduos tenham acesso a serviços de saúde de qualidade, os quais garantam uma assistência contínua e integral, em diferentes níveis de complexidade, exigidos no manejo da doença. É perceptível que a APS não esgota seus recursos terapêuticos, encaminhando uma demanda considerável de pacientes para a Atenção Secundária, a qual atua, muitas vezes, de maneira reducionista sobre o indivíduo, considerando apenas a doença (Corrêa et al., 2017).

As fontes de cadastros de doenças crônicas como o diabetes, quando preenchidas adequadamente, permitem que as unidades de APS organizem a continuidade do cuidado e monitorem os fatores de risco. Esta organização e intervenções clínicas, como programas educativos para a população em risco e para a população já diagnosticada com diabetes, podem modificar o panorama nacional e internacional de crescimento epidêmico, contribuindo para a qualidade de vida dos indivíduos (Cortez et al., 2015).

É importante reforçar que o DM, assim como as demais doenças crônicas, tem sua prevalência aumentada em pessoas acima de 50 anos de idade e tem crescido de forma significativa, sendo a doença crônica não transmissível que mais cresce, principalmente nos países em desenvolvimento. Esta realidade demonstra o processo de envelhecimento e hábitos poucos saudáveis adotados pela população brasileira como dieta inadequada e o sedentarismo. Ainda, caracteriza a transição demográfica 
e a necessidade dos profissionais da saúde arquitetarem planejamentos estratégicos e programas de atendimentos específicos às comorbidades e características sociodemográficas da sua população, oferecendo ao indivíduo maneiras de promover o controle metabólico (Cortez et al., 2015).

Considerando a importância de se identificar o conhecimento dos profissionais da APS sobre a educação em saúde, essa categoria analisou as considerações dos participantes acerca dessa temática. Segundo relatos, a educação em saúde pode ser identificada como uma estratégia que tem como finalidade prevenir e promover a saúde da população assistida (Barreto et al., 2019).

Investir na educação em saúde relacionada à alimentação saudável durante as consultas de DM é primordial para evitar alterações glicêmicas e manter o controle da doença, como também investir em atividades em grupos operativos, oficinas e palestras, considerando as características do indivíduo, como idade, escolaridade, tempo de diagnóstico, questões psicossociais e culturais (Marques et al., 2019).

O monitoramento foi uma das ações educativas realizadas de forma sistematizada a cada três meses (periodicidade), cuja finalidade consiste em melhorar os conhecimentos e as atitudes em relação à doença para o favorecimento da mudança de comportamento associada ao autogerenciamento dos cuidados e o controle da doença. Encontra-se entre seus objetivos específicos, em relação aos usuários: melhorar os conhecimentos, no que diz respeito à própria doença, a dieta e a prática de exercícios físicos; tomar consciência dos valores e atitudes relacionados aos aspectos psicológicos associados à doença; favorecer a mudança de comportamento para a adesão a dieta e atividade física; melhorar o controle clínico da doença (Torres et al., 2013).

Os usuários com DM constroem suas próprias estratégias para lidar com essa condição crônica, buscando outros saberes além dos científicos. Para eles, as práticas educativas precisam se configurar em um processo abrangente, não apenas incluindo informações sobre o tratamento tradicional, mas também proporcionando espaço apropriado para criar vínculos entre usuários e profissionais e proporcionar autonomia (David, Torres \& Reis, 2012).

Para o tratamento do DM são imprescindíveis a vinculação do paciente às unidades de atendimento, a garantia do diagnóstico e o atendimento por profissionais atualizados, uma vez que seu diagnóstico e controle evitam complicações ou, ao menos, retardam a progressão das já existentes. Além disso, o maior contato com o serviço de saúde promove mais adesão ao tratamento (Silva et al., 2014).

A associação encontrada entre o atendimento realizado pelo mesmo enfermeiro também está relacionada ao vínculo e acolhimento. Esses dois atributos contribuem para o desenvolvimento de ações de prevenção e promoção da saúde, favorecem o reconhecimento das necessidades dos usuários e, por conseguinte, promovem mais satisfação com o serviço. Destaca-se que o enfermeiro na equipe da ESF é o profissional que mais tem contato e conhecimento das condições de saúde da população atendida, não sendo incomum que ao longo do tempo estabeleça uma relação de confiança com o usuário, deixando-o mais à vontade para expor seus problemas e, muitas vezes, seguir as recomendações propostas (Santos et al., 2020).

\section{Conclusão}

O presente estudo demonstra que há a necessidade de manutenção do controle da diabetes mellitus nos pacientes atendidos na atenção primária visando o bem-estar de todos. Através da educação em saúde os profissionais conseguem promover a saúde a esses pacientes acometidos com essa doença e reduzir os riscos dessas complicações. Para isto, todos os profissionais da equipe precisam estar atualizados de acordo com os protocolos do Ministério da Saúde para uma boa adesão ao tratamento.

Diversos meios são utilizados para a redução de complicações graves a esses pacientes com DM como: tratamento medicamentoso, atividade física dentre outros meios que possam fazer com que esse paciente tenha uma boa qualidade de vida não tendo interferências na sua rotina, atividades e afazeres. 
Conclui-se com esse estudo de revisão integrativa, que é relevante para a literatura no tocante ao incentivo do conhecimento teórico-prático acerca da qualidade de vida de pacientes com diabetes mellitus na atenção primária.

Essa pesquisa contribui não somente para a formação acadêmica, mas sugere e estimula a produção de novas pesquisas de temática similar, que abordem a importância da qualidade de vida de pacientes com DM na atenção primária para que se possa contribuir futuramente na melhor qualidade de vida da sociedade em geral.

\section{Referências}

Araújo, M. F. M. D., Freitas, R. W. J. F. D., Fragoso, L. V. C., Araújo, T. M. D., Damasceno, M. M. C., \& Zanetti, M. L. (201 1). Cumprimento da terapia com antidiabéticos orais em usuários da atenção primária. Texto \& Contexto-Enfermagem, 20(1), 135-143.

Araújo, E. S. S., da Silva, L. D. F., Moreira, T. M. M., de Almeida, P. C., de Freitas, M. C., \& Guedes, M. V. C. (2018). Cuidado de enfermagem ao paciente com diabetes fundamentado na Teoria de King. Revista Brasileira de Enfermagem, 71(3), 1157-1163.

Assunção, S. C., Fonseca, A. P., Silveira, M. F., Caldeira, A. P., \& Pinho, L. D. (2017). Conhecimento e atitude de pacientes com diabetes mellitus da Atenção Primária à Saúde. Escola Anna Nery, 21(4), 1-7.

Azevedo, A. L. S. D., Silva, R. A. D., Tomasi, E., \& Quevedo, L. D. Á. (2013). Doenças crônicas e qualidade de vida na atenção primária à saúde. Cadernos de saúde pública, 29(9), 1774-1782.

Barreto, A. C. O., Rebouças, C. B. D. A., Aguiar, M. I. F. D., Barbosa, R. B., Rocha, S. R., Cordeiro, L. M., \& Freitas, R. W. J. F. D. (2019). Percepção da equipe multiprofissional da Atenção Primária sobre educação em saúde. Revista Brasileira de Enfermagem, 72(1), $278-285$.

Corrêa, K., Gouvêa, G. R., Silva, M. A. V. D., Possobon, R. D. F., Barbosa, L. F. D. L. N., Pereira, A. C., \& Cortellazzi, K. L. (2017). Qualidade de vida e características dos pacientes diabéticos. Ciência \& Saúde Coletiva, 22(3), 921-930.

Cortez, D. N., Reis, I. A., Souza, D. A. S., Macedo, M. M. L., \& Torres, H. D. C. (2015). Complicações e o tempo de diagnóstico do diabetes mellitus na atenção primária. Acta Paulista de Enfermagem, 28(3), 250-255.

David, G. F., Carvalho T., H., \& Reis, I. A. (2012). Atitudes dos profissionais de saúde nas práticas educativas em diabetes mellitus na atenção primária. Ciência, Cuidado e Saúde, 11(4), 758-766.

Faria, H. T. G., Veras, V. S., Xavier, A. T. D. F., Teixeira, C. R. D. S., Zanetti, M. L., \& Santos, M. A. D. (2013). Qualidade de vida de pacientes com diabetes mellitus antes e após participação em programa educativo. Revista da Escola de Enfermagem da USP, 47(2), 348-354.

Frota, S. S., Guedes, M. V. C., \& Lopes, L. V. (2015). Fatores relacionados à qualidade de vida de pacientes diabéticos. Rev Rene, 16(5), 639-648.

Marques, M. B., Coutinho, J. F. V., Martins, M. C., Lopes, M. V. D. O., Maia, J. C., \& Silva, M. J. D. (2019). Intervenção ed ucativa para a promoção do autocuidado de idosos com diabetes mellitus. Revista da Escola de Enfermagem da USP, 53, 1-8.

Moreschi, C., Rempel, C., Foggiato de Siqueira, D., Stein Backes, D., Felipe Pissaia, L., \& Quevedo Grave, M. T. (2018). Estratégias Saúde da Família: perfil/qualidade de vida de pessoas com diabetes. Revista Brasileira de Enfermagem, 71(6), 3073-3080.

Nakagaki, M. S., \& McLellan, K. C. P. (2013). Diabetes tipo 2 e estilo de vida: papel do exercício físico na atenção primária e secundária. Saúde em Revista, 13(33), 67-75.

Pereira, A. S., et al. (2018). Metodologia da pesquisa científica. UFSM. https://repositorio.ufsm.br/bitstream/handle/1/15824/Lic_Computacao_MetodologiaPesquisa-Cientifica.pdf?sequence $=1$.

Salci, M. A., Meirelles, B. H. S., \& Silva, D. M. G. V. D. (2018). Educação em saúde para prevenção das complicações crônicas do diabetes mellitus na atenção primária. Escola Anna Nery, 22(1), 1-6.

Santos, A. L., Marcon, S. S., Teston, E. F., Back, I. R., Lino, I. G. T., Batista, V. C., \& Haddad, M. D. C. F. L. (2020). Adesão ao tratamento de diabetes Mellitus e relação com a assistência na atenção primária. Revista Mineira de Enfermagem, 24, 1-10.

Santos, L., \& Torres, H. D. C. (2012). Práticas educativas em Diabetes Mellitus: compreendendo as competências dos profission ais da saúde. Texto \& ContextoEnfermagem, 21(3), 574-580.

Soares, D. A., Pereira, D. F., Rebouças, M. O., Oliveira, J. E., \& Santana, L.V. (2014). Adesão ao tratamento da hipertensão e do diabetes-compreensão de elementos intervenientes segundo usuários de um serviço de atenção primária à saúde. Revista de APS, 17(3), 311-317.

Silva, T. F. A. D., Rodrigues, J. E. G., Silva, A. P. S. M., Barros, M. A. R., Felipe, G. F., \& Machado, A. L. G. (2014). Con sulta de enfermagem à pessoa com diabetes mellitus na atenção básica. Revista Mineira de Enfermagem, 18(3), 710-716.

Torres, H. C., Reis, I. A., Roque, C., \& Faria, P. (2013). Monitoramento telefônico como estratégia educativa para o autocuidado das pessoas com diabetes na atenção primária. Ciência y Enfermeria, 19(1), 95-105.

Vietta, G. G., Volpato, G., Kretzer, M. R., da Gama, F. O., Nazário, N. O., \& Pereira, E. (2019). Impacto do conhecimento nas atitudes, no sofrimento e qualidade de vida do paciente diabético. Arquivos Catarinenses de Medicina, 48(4), 51-61. 
Research, Society and Development, v. 10, n. 5, e15610514999, 2021

(CC BY 4.0) | ISSN 2525-3409 | DOI: http://dx.doi.org/10.33448/rsd-v10i5.14999

Zulian, L. R., Santos, M. A. D., Veras, V. S., Rodrigues, F. F. L., Arrelias, C. C. A., \& Zanetti, M. L. (2013). Qualidade de vida de pacientes com diabetes utilizando o instrumento Diabetes 39 (D-39). Revista Gaúcha de Enfermagem, 34(3), 138-146. 